\title{
QALYs vs DALYs vs LYs gained: What are the differences, and what difference do they make for health care priority setting?
}

\author{
Bjarne Robberstad \\ Department of Public Health and Primary Health Care, Centre for International Health, University of Bergen \\ Correspondence: Bjarne Robberstad, Institutt for samfunnsmedisinske fag, UiB, Kalfarveien 31, NO-5018 Bergen, Norway \\ Telephone: +47 55586479 Telefax: +4755586130 E-mail: bjarne.robberstad@cih.uib.no
}

\begin{abstract}
SUMMARY
QALYs, DALYs and life years gained are all common outcome measures in economic evaluations of health interventions. While the latter is a pure measure of mortality, QALYs and DALYs are measures that combine mortality with morbidity in single numerical units, an exercise involving trade-offs between quantity for quality of health. Some authors have argued that mortality and morbidity are totally different dimensions, and combining them into a single numerical unit is nonsensious. Others have argued that the exercise is necessary in order to convert principles for resource allocation to criteria that can be used in a consistent manner. This paper has a two-fold objective, namely to discuss the differences between these health measures, and to explore what difference they are likely to make for health care priority setting in sub-Saharan Africa.
\end{abstract}

\section{INTRODUCTION}

The total population of Africa is some 655 million people, who experience an annual loss of about 358 million disability adjusted life years (DALYs). This is almost half a DALY per capita per year, with HIVAIDS, childhood diarrhoea, measles, malaria, and respiratory infections among the most important causes of disease burden (1). The life expectancy in the region is generally short, with 47 years in Tanzania and 37 years in Malawi as examples (2). Effective treatment or prevention is available for most of the above conditions (1), but health care budgets that are extremely scarce represent effective barriers against improved population health. Generally for the region, health care budgets are in the range 3-20 USD per capita per year, while for e.g. Tanzania the health budget in 2002 was 7 USD per capita (3). To put this into perspective, the public health care expenditures in Norway were 3366 USD per capita last year (3), almost 500 times higher.

In this situation, with health challenges that are immense and budgets that are extremely scarce, giving priority to the "wrong" programs may have large implications for health system performance. The opportunity cost of wasting 10 USD in a country like Tanzania is more than one year of human life. An example is immunisation against hepatitis $\mathrm{B}$, which is not routinely done in a majority of sub-Saharan African countries (4). This intervention has been demonstrated to cost as little as 8 USD per life year saved (5). Putting weight on cost-effectiveness seems like a good idea in this situation, because when resources are very scarce it seems reasonable to make the most of it. Economic evaluation, like cost-effectiveness analysis (CEA), has therefore been assigned a key role since the World Development Report 1993 (6).
For CEA to serve a meaningful role in comparing the relative value of different health interventions, it is necessary to apply a commonly agreed measure of population health. The objectives of this paper are therefore to present the major differences between three commonly applied health measures; life years gained (LYs gained), quality adjusted life years (QALYs) and disability adjusted life years (DALYs). Furthermore, it will be explored what difference these alternative measures may have for health care priority setting in sub-Saharan Africa.

\section{LIFE YEARS GAINED}

Traditionally, the impact of health care has been measured in terms of its effect on mortality (7), for example deaths averted. A potential drawback of using deaths averted to measure health effects is that the method doesn't distinguish between children, adults and the elderly. This is not unproblematic since most people, if forced to make a choice, would prefer to save e.g. a 5-year-old infant rather than a person aged 95.

Life Years gained is a modified mortality measure where remaining life expectancy is taken into account. This method accrues more weight to young target populations, because saving the life of an infant yields more life years than saving the life of an old person. Life years are calculated as the remaining life expectancy at the point of each averted death. Life expectancies may be taken from life tables that are specific for each setting or standardized across larger regions. The choice of life table is not uncontroversial, as a life table with high life expectancies will yield more life years and render interventions more attractive than life tables with shorter life expectancies. 


\section{DisCOUNTING OF HEALTH EFFECTS}

LYs gained can be discounted, to adjust for time differences, or presented undiscounted. Common practice is to discount health outcomes at a rate between 3 and $5 \%(8,9)$. Discounting accrues less weight to future than to immediate health outcomes, which is illustrated in Figure 1. With a 3\% discount rate, for example, half the weight is accrued to outcomes 23 years into the future compared to immediate outcomes, while a $5 \%$ discount rate reduces the weight to half in about 14 years. There is a relatively extensive amount of literature discussing whether or not the practice of discounting future health effects is appropriate. For example, discounting has been criticized for discriminating future generations and for being immoral (10). Others claim that for the sake of consistency, discounting of health as well as costs is necessary $(11,12)$. This debate will not be pursued further in this paper, because discounting is not what makes LY, QALYs and DALYs different. Nevertheless, the choice of discount rate is important because it may determine the mix between typical curative interventions, like improved case management of malaria, and typical preventive interventions, like hepatitis $\mathrm{B}$ vaccination.

\section{AdVAnTAges AND DiSAdVANTAges OF LY GAINED}

LYs saved is as indicated a relatively easy and transparent method for measuring population health, and there are few value choices involved. The perhaps most important disadvantage is that the method ignores the obvious fact that health is more than merely staying alive. The method will fail to acknowledge health improvements such as improved physical ability, reduced neuropsychological stress, and reduced chronic pain. This makes it difficult to compare outcomes of health interventions that target e.g. chronic conditions with interventions targeting fatal conditions. Furthermore, if only the mortality aspect is quantified and included in the denominator of the cost-effectiveness ratio, LY gained represent an intrinsic bias against conditions that are largely non-fatal like for example poliomyelitis, onchocercosis (river blindness) and schizophrenia.

\section{TAKING MORBIDITY INTO ACCOUNT}

It is non-controversial that it is necessary to say something about the majority of people who do not suffer premature death (13). Albert Schweitzer once said that "Pain is a more terrible lord of mankind than even death itself" (14). It is therefore, at one level or another, necessary to take people's morbidity into account. This recognition has led to the development of measures that simultaneously seek to capture gains from reduced morbidity and reduced mortality. The two dominating such summary measures are the Quality Adjusted Life Years (QALYs), and the Disability Adjusted Life Years (DALYs). QALYs and DALYs represent an implicit trade-off between quantity for quality of well-being. Such trade-offs are well known from many aspects of life, but many are unfamiliar with the concept of trading off quality for quantity of years of life and health.

William Somerset Maugham, in the novel "As a lily in the field", describes a young banker in London (Thomas Wilson) who found the prospective of doing the same kind of work, day by day, for the rest of his life rather depressing. Thomas Wilson once went on a holiday to Capri in Italy, and later he kept daydreaming about the swimming, the wine yards, the hillsides in moonlight, the sea and piazza in the evening. At the age of 35 he quits his job, sells his house and uses all his assets to invest in a life pension. The life pension will provide him with sufficient means to

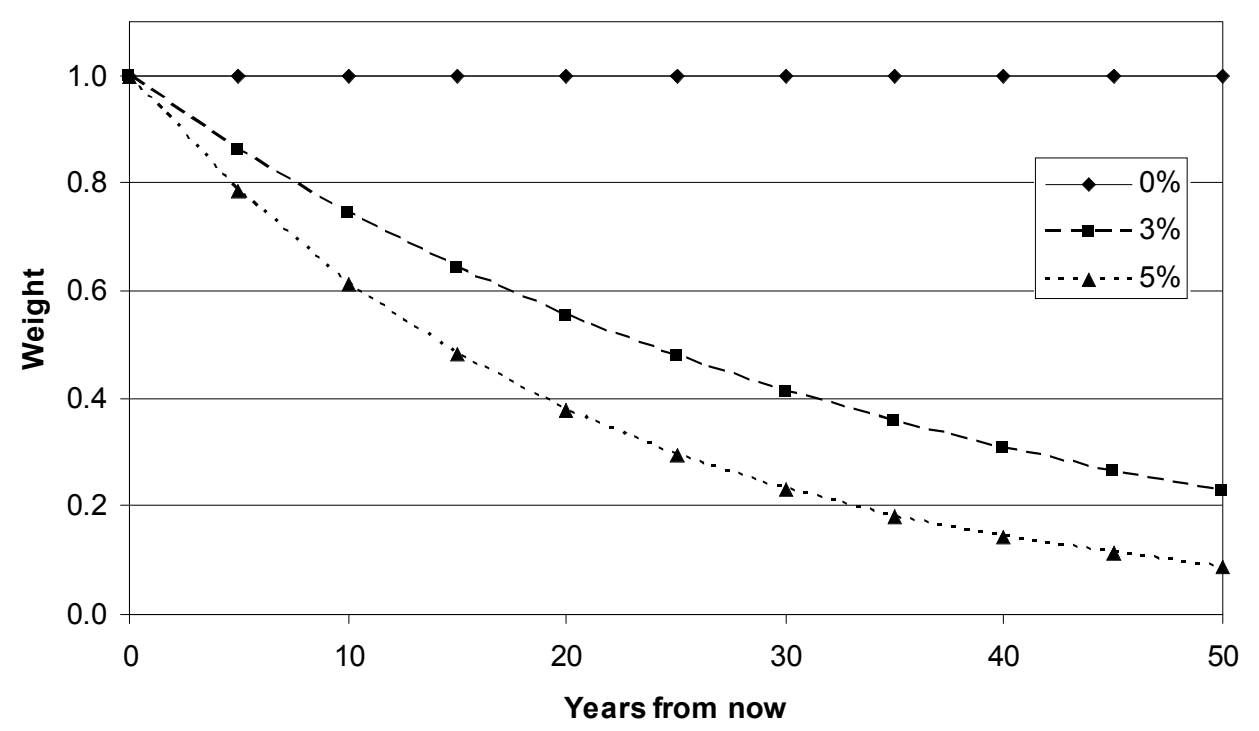

Figure 1. The effect of discounting on the weight of future health effects. 
rent a house at Capri, employ a servant to tend for him, enough for tobacco, good food, and a book from time to time for a period of 25 years. Rather than living, in his view, a miserable life as a banker for the remaining life time, he decides to live like "a lily in the field" for 25 years, till he is 60 , and then put an end to it. In other words, Thomas Wilson makes a plan that enables him to trade-off a long and miserable life for a shorter but qualitatively superior life.

\section{Quality AdJusted Life Years (QALYs)}

The story of Thomas Wilson brings us to the QALY, which was first introduced by Klarman et al. (15), although the concept is more commonly associated with an applied paper by Weinstein and Stason (16). In QALYs, premature death is combined with morbidity by attaching a weight to each health state such that value 0 represents death, while value 1 represents full health. The number of QALYs for a health profile is found by multiplying the health related quality of life weight (HRQoL) of the health state, with the duration of the health state. Figure 2 illustrates a person who gets some disability at the age of 10 , lives with the condition for 35 years, and suffers premature death at the age of 45 . If the life expectancy is 60 years, and the health related quality of life weight associated with the condition is 0.75 , the (undiscounted) lifetime QALYs of this person are $1.0 \times 10$ (QALYs before onset of disease) $+0.75 \times 35$ (QALYs during disease) $=36.25$. The QALY loss would be $1.0 \times 60$ (QALYs in case of full health for full life expectancy) $-36.25=23.75$. Like the LY measure, QALYs can be presented discounted or undiscounted, although the former is most common.

\section{Disability AdJusted Life Years (DALYs)}

The DALY concept was introduced by the World Bank in the World Health Report 1993 (6). Two important propositions are underlying the DALY concept, namely (i) that the burden calculated for like health outcomes should be the same, and (ii) that the non-health characteristics that should be considered should be restricted to age and sex (14). Other characteristics, like income, education, ethnicity, etc. should in other words not be taken into account.

These propositions represent intensions of creating a methodology that treat people as equal as possible. However, some would say that more weight should be put on the diseases of the disadvantaged, and that failing to do so is inequitable. Anand and Hanson, for example, claims that disease weights should take account of the way in which individual and social resources can compensate for the level of disability experienced (17). For example, being blind in Niger is worse than being blind in the UK, because the structural interventions in the UK make the disability less severe than in Niger (18). Blindness, according to this view, should therefore be weighted more heavily in Niger than in the UK.

Like the QALY, the DALY measure facilitates comparisons of all types of health outcomes by attaching disease weights were value 0 represents full health and value 1 represents death. Note that these disease weights are the opposite of the HRQoL weights in the QALY. A DALY can therefore be seen as an inverse QALY. Figure 2 illustrates that while QALYs are a good, DALYs are something one seek to avert. In the following section the statement that a DALY is an inverse QALY will be somewhat modified because of different weighting and elicitation techniques.

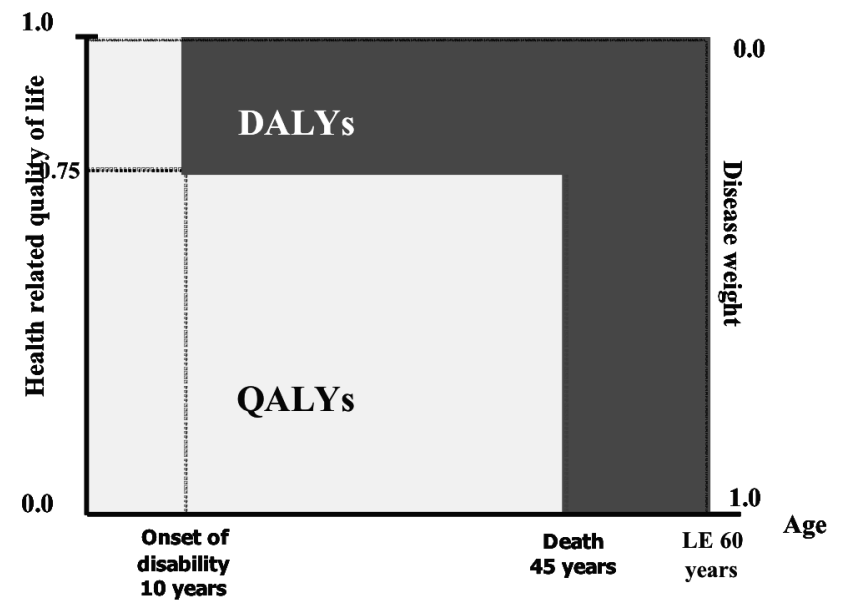

Figure 2. Relationship between QALYs and DALYs when the DALYs are not age-weighted.

\section{WEIGHTING OF HEALTH OUTCOMES}

In the above section it has been shown that the different health measures LY, QALYs and DALYs are weighted in different ways. They are all adjusted for the life expectancy of people affected, giving more weight to the young. Furthermore, they are all usually discounted, giving more weight to immediate over distant outcomes. However, only DALYs and QALYs are weighted to account for disease severity in order to summarise mortality and morbidity. The following section will partly be devoted to explaining how such weights can be elicited. Furthermore, DALYs are socalled age-weighted, and this also calls for some explanation. Finally, it has been proposed that such health measures should incorporate that extra weight or priority should be given to the most severely ill. This last concept has yet not been implemented in any of our outcome candidates in applied research, but the argument has some intuitive appeal, and at the end of this section some advantages and problems with such severity weighting will briefly be discussed. 


\section{ELICITATION OF WEIGHTS}

Several methods are being used to elicit health related quality of life or disability weights. The simplest approach is the so-called Visual Analogue Scale (VAS), where respondents are asked to rate health states on a scale. This scale often has the range from zero to one, where zero is the worst possible outcome (assumed to be death), and one is the best possible (perfect health). VAS is a simple method, but has been strongly criticised for not being choice based, in other words, that it does not involve a trade-off between quantity for quality of years.

In the time trade-off (TTO) methodology people are asked to choose between two certain alternatives involving trade-off between quantity and quality of life. For example, they may be asked how many years of perfect health they would consider equally good as living 10 years with psoriasis. TTO questions is the most common technique for elicitation of HRQoL weights in QALYs, although VAS and standard gamble (see below) are also sometimes used.

Person trade-off (PTO) is structurally similar to TTO, but here people are asked to trade off extending the lives of people with full health vs improving the health expectancy of people with some disability from sub-optimal to perfect health. This method was used to elicit disease weights for the DALY methodology from a panel of health experts, while the HRQoL weights in QALYs are usually found by interviewing lay people and patients.

In the standard gamble (SG) approach, respondents are asked to choose between the certainty of an intermediate health state, and the uncertainty of a treatment with two possible outcomes, where one of the outcomes is more attractive than the certain outcome, and the other is less attractive (e.g. death). The inclusion of uncertainty makes SG more consistent with standard economic utility theory than the other methods, but it is also probably the cognitively most demanding of the techniques for respondents to answer.

Disease weights or HRQoL weights may easily influence priority decisions. It is therefore of interest whether or not the choice of elicitation technique is likely to influence the weights. From a theoretical point of view, one may expect that weights elicited with SG are higher than the VAS because of risk aversion. One may also expect TTO weights to be higher than VAS weights because of time preferences.

These expectations were confirmed by Salomon and Murray, who in a sample of 69 individuals found that PTO weights were higher than SG, which again were higher than TTO, while weights produced by VAS were the lowest (19). In other words, VAS seemed to weight conditions as more severe than the other alternatives. While there was significant disagreement between the methods on the level of severity in this study, there was, however, considerable agreement in the rank orderings of the 12 conditions
(19). Currently, there is no agreement on which method that should be preferred for eliciting disease or quality of life weights $(19,20)$.

\section{Age Weighting}

Age weighting is a concept unique to the DALY methodology, and is intended to account for the fact that people are supported by others during infancy and at an advanced age, but support others during adulthood. This notion is called welfare interdependence. Welfare interdependence does not imply that the time lived at different ages are more or less important to those individuals, but that the social value is different (14).

The age weighting of DALYs therefore accrue more weight to the life years of bread-feeders and care takers, and can be illustrated by a hump-shaped curve which starts at zero for newborns, peaks at 25 , and gradually declines throughout adulthood without ever reaching zero. It can be argued that welfare interdependence is an arbitrary choice for an age-weighting function. In fact there exists several alternative ways to specify age weights depending on different perceptions on fairness. I will mention two alternatives to the DALY age-weights.

The first alternative is the so-called simple utilitarian ageism $(21,22)$, or horizontal age-weights. Horizontal age-weights accrue equal weight to all years, and is what is being used in the traditional QALY measure and non age-weighted DALYs. The last alternative is funded on the fair innings argument (23), and is called egalitarian ageism $(22,24)$. According to this argument everyone is entitled to a similarly long and healthy life, and the implication is that anyone failing to achieve this has been cheated, while anyone getting more is living on borrowed time (25). The elderly, according to this argument, have already had their fair share of life, and should be given gradually less weight in the distribution of scarce health care resources. These three alternative age-weighting functions are illustrated in Figure 3, but it should be noted that several sub-versions of the principles exists (21).

Tsuchia has reviewed the empirical evidence on age weights, and sums up that (i) there is no support for a horizontal age weight profile (current QALY practice) and (ii) there is broad agreement that the profile declines after middle age, but (iii) there is some disagreement on whether middle age or childhood is the peak (22). Despite the agreement that young should have some priority over the old, as Williams points out, the consensus is likely to evaporate when the quantitative issue is addressed as to how much priority the young should have (25).

\section{EQUITY WEIGHTING}

There is considerable empirical evidence suggesting that people in general are inequality averse in the sense that they hold the view that more weight should be 


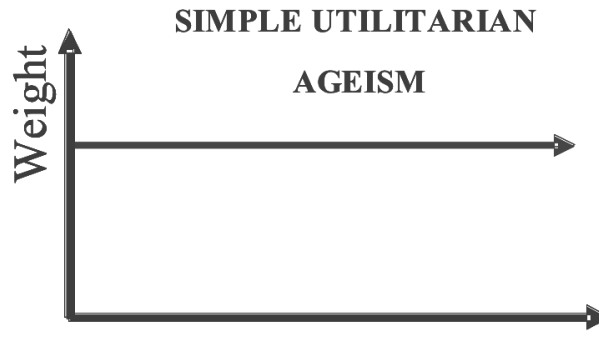

Age

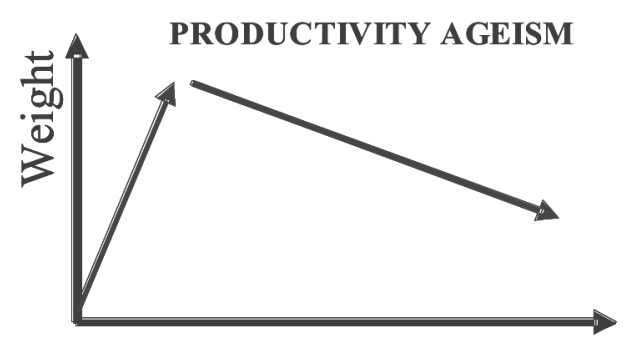

Age

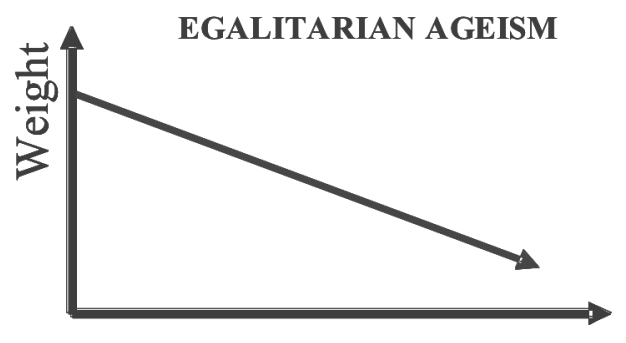

Age

Figure 3. Three alternative age-weighting functions.

allocated to those that are most severely ill. However, equity weighting is at current not standard procedure neither in DALYs, nor QALYs or LYs gained. Introduction of equity weighted QALYs has by Alan Williams been proposed as "...the best way to integrate efficiency and equity in health care" (25). And furthermore that equity weights "...rules out the giving of priority to things that do no good, which is the danger with equalization policies". Williams argued for a version of the fair innings, where the quality of a person's life is important as well as the length of it (25).

There are two major barriers to implementing equity weighted QALYs or DALYs. First, there exists no agreement as to over which notion of disease severity one should pursue equity. Is it for example acute ill-health, or is it life time ill-health (26)? Secondly, empirical evidence including both estimation of equity weights and implementation of them in QALY maximization exercises is scarce. Bleichrodt and colleagues estimated equity weights in two Dutch populations. When they incorporated these weights in QALY calculations, they found that this in fact changed the ranking of 12 health interventions (27). These findings suggest that the issue of equity weighting need further research, although it is likely that such an enterprise will be quite data hungry (25).

\section{WHAT DIFFERENCE DO THEY MAKE?}

After having briefly presented some major differences between LYs gained, QALYs and DALYs, it is time to approach the second objective of this paper; What difference do they make for health care priority setting? I will first address the question by looking at a small selection of the literature in the area. For example, it has been argued that quality adjustment of life years makes little difference for priority setting. Others have argued that indeed it makes a difference, and moreover, that the difference is unfair or discriminating. Secondly, I will address the question by looking into two applied cases that I know well, namely my own studies on the cost-effectiveness of zinc as adjunct therapy in childhood diarrhoea (28) and on pharmaceutical preventive cardiology (29).

\section{"QUALITY ADJUSTMENTS MAKES LITTLE DIFFERENCE"}

Chapman and colleagues concluded that quality adjustments makes little difference for priority setting after having considered 63 CEAs reporting both costs per QALY and costs per LY gained. They therefore concluded that in most cases findings can be reported as costs per LY rather than the technically more challenging costs per QALY (30). This conclusion is partly based on the finding that quality adjustment of life years did not substantially change the estimated level cost-effectiveness. More specifically, they found that switching from QALY to a pure mortality measure like LYs gained altered the conclusion of whether or not to support implementation of health programs only in $18.5 \%$ of the cases (30). This is of course interesting, but if one believes that morbidity should be taken into account, it may also be interpreted as a simple measure like LY gained leading to the wrong conclusion in almost one fifth of the cases.

Furthermore, Chapman and colleagues investigated the number of times the ratio difference (Costs/LY Costs/QALY) was positive, zero or negative. They found that $60 \%$ of the ratio differences were positive (30). A positive ratio difference is the expected finding and implies that the cost-effectiveness ratios become more favourable when life years are quality adjusted. Furthermore, they found that $3 \%$ of the ratio differences were zero, implying that morbidity is not an issue for these conditions, while $36 \%$ of the ratio differences were negative (30). The latter of these observations means that quality adjustment has reduced the denominator in the cost-effectiveness ratio. This is conceivable only in two situations; (i) when a treatment has side effects affecting the quality of life sufficiently to out-crowd its life extending effect (e.g. cancer treatment when prognosis is poor), or (ii) when the health improvement of a treatment is out-crowded by shortened life expectancy. The main lesson, nevertheless, seems to be that quality adjustments increase the 
attractiveness of interventions in terms of costeffectiveness, and therefore increase the likelihood of implementation, all other things being equal.

\section{"DALY WEIGHTS ARE UNFAIR"}

There have been some concerns on whether or not the weighting of DALYs is fair. With exception of the age-weights, this critique also applies to QALYs. Anand and Hanson have claimed that "Using the DALY framework for resource allocation may lead to consequences that are at odds with principles of equity". They are worried that life-extension of disabled people avert fewer DALYs, and therefore count less, than life-extension of able-bodied people (17). Hypertension management of people with polio would, for example, avert fewer DALYs than hypertension management of people without such a chronic condition because each life year gained will be given less weight. The disability weight of polio is 0.369 (14).

This critique seems to be formally correct, but incorrectly applied. When DALYs, or QALYs, are used to estimate burden of disease, or in cost-effectiveness analysis to inform resource allocation, no such distinction is made between disabled and able-bodied people. The cost-effectiveness of e.g. preventive cardiology is done assuming that people have none unrelated conditions, like polio. The critique of Anand and Hanson therefore does not seem to apply to DALYs and QALYs the way they are used in economic evaluations.

Anand and Hanson are also critical to ageweighting, and argue that there are no reasons for valuing time lived at different ages differently, and that a principle of universalism would argue strongly for a common intrinsic valuation of human life, regardless of the age at which it is lived (17). Others are less worried by this kind of discrimination, because of the fact that we may all experience each age level. The discriminatory effect of setting unequal age weights is therefore ethically very different from the setting of weights according to ethnicity, sex, education or income (22).

\section{"DALY WEIGHTS ARE DISCRIMINATING"}

It has been argued that disability weights are discriminating developing countries. In the DALY manual, disability weights for clinical conditions are reported both before and after treatment, based on the general idea that a disease is less burdensome if it is treated. In a paper exploring whether DALY weights can influence global priority setting, Arnesen and Kapiriri claim that since few of the communicable diseases, which are typical developing country conditions, are reported with reduced disease weights after treatment, the DALY methodology is discriminating developing countries (31). Arnesen and Kapiriri are also critical to the general level of disease weights between commu- nicable and non-communicable diseases, indicating that the non-communicable diseases generally should have been weighted more heavily (31).

Furthermore, Arnesen and Kapiriri claim that disease weights discriminate against the young, because non-communicable diseases, which are a relatively higher burden in the elderly population, generally are assigned higher weights than the communicable diseases, which are relatively more important in young populations. In particular, they question that a condition like developmental disability due to malnutrition, primarily affecting children, has a disease weight of only 0.024 , while unipolar major depression, which is a typical adult condition, has a weight of 0.600 (31). The young are also discriminated, in their view, because of the age-weights (31).

It is evidently correct that the disease weights of the DALY methodology needs to be looked further into, with respect both to elicitation techniques, level of disease burden and change of weights after treatment. Several examples given by Arnesen and Kapiriri illustrate this, for example that amputation of a thumb has been assigned higher disease weight than amputation of an arm (14,31). Without further empirical evidence, there are few reasons neither to support nor to dismiss Arnesen and Kapiriri's general critique of the level of disability weights for different groups of diseases. Nevertheless, it is a main point that since health care resources are generally distributed within and not between countries, the claim that people with communicable diseases and developing countries are discriminated due to disease weighted DALYs remains undocumented.

Arnesen and Kapiriri also conclude that ageweights lead to the disease burden carried by the youngest age groups are given considerably less weight, and hence, less priority than it would without these choices (31). This conclusion is true, but only in the case of non-fatal and non-chronic conditions. For fatal or chronic conditions, on the other hand, it can be demonstrated that age-weights favour people aged less than 35-45 years. The reason for this is that for fatal or chronic conditions, the DALY calculations of young people will include the peak of the hump shaped ageweighting function. This is in fact illustrated in Figure 1 in Arnesen and Kapiriri's own paper (31), where different combinations of discounted/undiscounted and age-weighted/non age-weighted DALYs are presented. However, the authors fail to sufficiently pull forward this finding and base their conclusion on the joint effect of age weighting and discounting, which in fact pulls in opposite directions with respect to the burden on the youngest. The aggregate effect of age-weights on children, including chronic, recurrent, fatal and non-fatal conditions, remains uncertain. However, if we believe that mortality is more important for burden of disease than morbidity, age-weights will lead to more resource being allocated to interventions targeting children. 


\section{THE EFFECT OF WEIGHTING IN TWO APPLIED ECONOMIC EVALUATIONS}

From the above theoretical considerations, it is useful to turn our attention towards two applied economic evaluations and consider how disability weighting, age-weighting and discounting influence the costeffectiveness and attractiveness in these cases. The scenarios of these two studies are different over several dimensions, and therefore useful to illustrate what difference different outcome measures may have for health care priority setting. The first of these is an economic evaluation of zinc as adjunct therapy in diarrhoea (28), while the second is a study of the costeffectiveness of pharmaceutical interventions to prevent cardiovascular disease in sub-Saharan Africa (29).

Both of the two economic evaluations include a number of treatment alternatives. In Table 1, the costeffectiveness ratios (CERs) for two arbitrary example interventions are presented, zinc and oral rehydration solution in diarrhoea management, and aspirin and a diuretica given to patients with very high cardiovascular risk. Results are first presented as USD per LY gained, secondly as USD per non age-weighted DALY, which roughly corresponds to QALYs, and finally as USD per standard age-weighted DALY. In all cases, both costs and health effects are discounted using a rate of $3 \%$.

Table 1. The impact on the cost-effectiveness ratio of using LY gained, QALYs (non-age-weighted DALYs) and standard age-weighted DALYs in interventions targeting childhood diarrhoea and preventive cardiology.

\begin{tabular}{lcc}
\hline & $\begin{array}{c}\text { Zinc in } \\
\text { childhood } \\
\text { diarrhoea }\end{array}$ & $\begin{array}{c}\text { Aspirin and diure- } \\
\text { tica in preventive } \\
\text { cardiology }\end{array}$ \\
\hline USD per LY gained & 74 & 55 \\
USD per DALY (no age-weights) & 70 & 46 \\
USD per DALY (age-weighted) & 66 & 56 \\
\hline
\end{tabular}

The CER of zinc is 74 USD per LY gained, which improves slightly to 70 USD per non-age-weighted DALY (or QALY) by including a disease weight of 0.119 for diarrhoea. When the outcome in addition is age-weighted to standard DALYs, the CER further improves to 66 USD per DALY (see Table 1). In the case of diarrhoea, it therefore turns out that both disease weighting and age weighting makes the intervention more cost-effective and therefore more attractive for implementation. This result supports the view that age weighting is likely to favour interventions targeting children.

While diarrhoea is a disease group typically affecting young children, preventive cardiology is foremost relevant for adults aged 40 and higher. In this case, the CER improved from 55 USD per LY to 46 USD per non-age-weighted DALY (or QALY) by including disease weights of 0.268 and 0.490 for stroke and coronary heart disease, respectively. This improvement is rather large because the disease weights for stroke and coronary heart disease are quite high and because people who survive such attacks can be expected to live with a disability for their remaining lives. Inclusion of the morbidity component therefore makes a larger difference in cardiology than in diarrhoea, where each case has an expected duration of only 7 days (28) and the disease weight is relatively small.

While age weighting made diarrhoea management more attractive, the opposite is the case for preventive cardiology. For this intervention, the positive effect of disability weighting is out-crowded by age-weighting resulting in a CER of 56 USD per DALY. This is because cardiovascular patients are relatively old and already have passed the "hump" of the age-weight curve, and are facing declining age-weights for the remaining life span. This supports the undisputed view that age-weights are disfavouring the elderly.

\section{CONCLUDING REMARKS}

In this paper some of the differences between the health measures life years gained, QALYs and DALYs are briefly presented. To wrap up, one main difference is that whereas LY gained is a pure measure of mortality, the two latter seeks to incorporate morbidity by doing disability- and quality of life weighting. A major difference between QALY and DALY is that the latter measure in addition incorporates age-weights.

It has also been discussed what difference these alternative measures of health improvement may have for priority setting. Claims that health related quality of life weighting has little impact on priority setting have been briefly presented and discussed. Furthermore, worries that DALY weights are unfair and discriminating have been briefly presented. Some of the critique is evidently reasonable, in particular there seems to be a need to further improve and justify the DALY disease weights. Other parts of the critique are in my opinion undocumented, in particular the claim that age-weights discriminate children.

It is undoubtedly true that age weighting and disease weighting have ethical implications that are challenging, as critics frequently have pointed out. We have for example seen that the method used to elicit disease weights may influence the morbidity estimates, with visual analogue scales (VAS) seemingly weighting conditions as more severe than the other methods. If comparison is made across diseases where different elicitation techniques for disease severity have been used, this may lead to erroneous priority setting decisions. The direction of this error is, however, depending on the circumstances in each case.

The important question is not whether these weights and value choices may influence resource allocation. Because clearly, making a difference is what 
they are intended to do. A weighting principle that doesn't create winners, as well as losers, is inconceivable. One must therefore ask whether the weights brought about by QALYs and DALYs are contributing to improved population health and reduced health inequalities in sub-Saharan Africa.

All the measures - LYs, QALY and DALY clearly lead to more weight being accrued to the young over the elderly, compared to a simple measure of mortality like deaths averted. This is in accordance with both an objective of improved population health and common notions of equality. The effects on population health and health inequalities of moving from LYs gained to QALY or DALY are perhaps less obvious. It might be argued that in settings with extreme resource scarcity with many conditions that can be treated cheaply and effectively, crude estimates of population health are sufficient to inform priority setting. It may further be argued that more subtle analysis, such as the QALY and DALY will not give more real information than such crude measures. Many doubt that it at all is possible to translate fatal and nonfatal health outcomes into one numerical unit. How, asks Hilda Bastian, do you decide which problems and limitations are worse than others, and give them a numerical weight (32)? Arnesen and Kapiriri worries that valuable epidemiological information may lose importance if it mixed with value choices of unknown validity (31). Taken together, such concerns cast doubt on the value of summary health measures like the QALY and DALY in improving population health.

On the other hand, the number of even basic health services that cannot be financed in settings with extreme resource scarcity is very large. This, I believe, increases the importance of developing and applying methodology that is as objective and consistent as possible. Obviously, there are challenging value choices embedded in these methods, but trade-offs between quantity for quality of health needs to be made in public health systems, they are in fact being made, and they will continue to be made, with implicit or explicit value judgements. The advantage of health outcomes combining mortality and morbidity, like the DALY, is that they can be used to inform such trade-offs in a fairly transparent and consistent way. Many feel that only trough quantification of morbidity, as well as mortality, it is possible to have a fair amount of resources allocated to rehabilitation of chronic conditions and marginal patient groups (33).

Irrespective of these views, in order for the policy mix to be perceived fair (equitable) as well as effective in improving population health within limited budgets, a continuous public scrutiny and evaluation of the applied weights and value choices needs to be made (34).

\section{REFERENCES}

1. WHO. World Health Report 2002. Geneva: World Health Organization, 2002.

2. Lopez AD, Ahmad OB, Guillot M, Inoue M, Ferguson BD, Salomon JA. Life Tables for 191 countries for 2000: Data, Methods, Results. Geneva: Evidence and Information for Policy (EIP). World Health Organisation, 2001. GPE Discussion Paper No. 40.

3. WHO. World Health Report 2005. Geneva: World Health Organization, 2005.

4. who.int [Homepage on the internet]. World Health Organization, 2004. Vaccines, Immunization and Biologicals. (Accessed 09.11.2004, at www.who.int/vaccines-surveillance/StatsAndGraphs.htm).

5. Miller MA, McCann L. Policy analysis of the use of Hepatitis B, Haemophilus Influenzae type B, Streptococcus pneumoniae-conjugate and rotavirus vaccines in national immunization schedules. Health Econ 2000; 9: 19-35.

6. WorldBank. World Development Report 1993: Investing in health. New York: Oxford University Press, 1993.

7. McKeown T. The direction of medical research. Lancet 1979; 2 (8155): 1281-4.

8. Smith DH, Gravelle $\mathrm{H}$. The practice of discounting in economic evaluation of health care interventions. Int $J$ Technol Assess Health Care 2001; 17: 236-43.

9. Walker D, Kumaranayake L. Allowing for differential timing in cost analyses: discounting and annualization. Health Policy Plan 2002; 17 (1): 112-8.

10. Parfit D. Reasons and Persons. Oxford: Clarendon Press, 1984.

11. Viscusi WK. Discounting health effects for medical decisions. In: Sloan FA, ed. Valuing Health Care Costs, Benefits, and Effectiveness of Pharmaceuticals and Other Medical Technologies. Cambridge: Cambridge University Press, 1995: 125-47.

12. Weinstein MC, Stason WB. Foundations of cost-effectiveness analysis for health and medical practices. $N$ Engl J Med 1977; 296: 716-21.

13. Dolan P. The measurement of health-related quality of life for use in resource allocation decisions in health care. In: Culyer AJ, Newhouse JP, eds. Handbook of health economics. Amsterdam: Elsevier, 2000: 1723-60. 
14. Murray C, Lopez A. The Global Burden of Disease. A Comprehensive assessment of mortality and disability from diseases, injuries, and risk factors in 1990 and projected to 2020: The Harvard School of Public Health, 1996.

15. Klarman H, Francis J, Rosenthal G. Cost-effectiveness analysis applied to the treatment of chronic renal disease. Med Care 1968; 6 (1): 48-54.

16. Weinstein MC, Stason WB. Hypertension: A Policy Perspective. Cambridge: Harvard University Press, 1976.

17. Anand S, Hanson K. Disability-adjusted life years: a critical review. J Health Econ 1997; 16 (6): 685-702.

18. Reidpath D, Allotey PA, Kouame A, Cummins RA. Measuring health in a vacuum: examining the disability weight of the DALY. Health Policy Plan 2003; 18 (4): 351-6.

19. Salomon JA, Murray CJL. A multi-method approach to measuring health-state valuations. Health Econ 2004; 13: 281-90.

20. Nord E. Cost-Value Analysis in Health Care. Cambridge: Cambridge University Press, 1999.

21. Tsuchiya A. QALYs and ageism: Philosophical theories and age weighting. Health Econ 2000; 9: 57-68.

22. Tsuchiya A. Age-related preferences and age weighting health benefits. Soc Sci Med 1999; 48: 267-76.

23. Harris J. QALYfying the value of life. J Med Ethics 1987; 13: 119-20.

24. Nord E, Street A, Richardson J, Kuhse H, Singer P. The significance of age and duration of effect in social evaluation of health care. Health Care Anal 1996; 4 (2): 103-11.

25. Williams A. Intergenerational equity: An exploration of the "fair innings" argument. Health Econ 1997; 6: $117-32$.

26. Cookson R, Dolan P. Principles of justice in health care rationing. J Med Ethics 2000; 26: 323-9.

27. Bleichrodt H, Doctor J, Stolk E. A nonparametric elicitation of the equity-efficiency trade-off in cost-utility analysis. J Health Econ 2005; 24 (4): 655-78.

28. Robberstad B, Strand T, Black RE, Sommerfelt H. Cost-effectiveness of zinc as adjunct therapy for acute childhood diarrhea in developing countries. Bull WHO 2004; 82 (7): 523-31.

29. Robberstad B, Hemed Y, Norheim OF. Cost-effectiveness of medical interventions to prevent cardiovascular disease in a sub-Saharan African country - the case of Tanzania. In: Robberstad B. Economic evaluation of health interventions in sub-Saharan Africa. Applied economic evaluations and studies on time preferences for health in Tanzania. Bergen: Dissertation for the PhD degree, University of Bergen, 2005.

30. Chapman RH, Berger M, Weinstein MC, Weeks JC, Goldie S, Neumann PJ. When does quality-adjusting life-years matter in cost-effectiveness analysis? Health Econ 2004; 13: 429-36.

31. Arnesen T, Kapiriri L. Can value choices in DALYs influence global priority setting? Health Policy 2004; 70 : $137-49$.

32. Bastian H. A consumer trip into the world of the DALY calculations: An Alice in Wonderland experience. Reprod Health Matters 2000; 8 (15): 113-5.

33. Jelsma J, Weerdt Wd, Cock Pd. Disability Adjusted Life Years (DALYs) and rehabilitation. Disabil Rehab 2002; 24 (7): 378-82

34. Sen A. Development as freedom. Oxford: Oxford University Press, 1999. 